\title{
Conformally soft theorem in gauge theory
}

\author{
Monica Pate $\odot$, Ana-Maria Raclariu $\odot$, and Andrew Strominger \\ Center for the Fundamental Laws of Nature, Harvard University, Cambridge, Massachusetts 02138, USA
}

(Received 16 August 2019; published 24 October 2019)

\begin{abstract}
Asymptotic particle states in four-dimensional celestial scattering amplitudes are labeled by their $S L(2, \mathbb{C})$ Lorentz/conformal weights $(h, \bar{h})$ rather than the usual energy-momentum four-vector. These boost eigenstates involve a superposition of all energies. As such, celestial gluon (or photon) scattering cannot obey the usual (energetically) soft theorems. In this paper we show that tree-level celestial gluon scattering, in theories with sufficiently soft UV behavior, instead obeys conformally soft theorems involving $h \rightarrow 0$ or $\bar{h} \rightarrow 0$. Unlike the energetically soft theorem, the conformally soft theorem cannot be derived from low-energy effective field theory.
\end{abstract}

DOI: 10.1103/PhysRevD.100.085017

\section{INTRODUCTION}

Four-dimensional (4D) Minkowskian scattering amplitudes are usually presented in a momentum-space basis, in which translations act trivially by phases, while the $S L(2, \mathbb{C})$ action of the Lorentz group is more nontrivial. The latter acts as the conformal group on the celestial twosphere at null infinity. Recently there has been interest in and explicit construction of amplitudes in a conformal basis on the celestial sphere [1-16], in which the conformal action is trivial but translation invariance is hidden. We will use the symbol $\tilde{\mathcal{A}}$ to denote such amplitudes (and distinguish them from the usual amplitudes denoted $\mathcal{A}$ ) and simply refer to them as celestial amplitudes. The structure of celestial amplitudes has so far turned out to be surprisingly rich. Properties which are obscure in $\mathcal{A}$ are sometimes obvious in $\tilde{\mathcal{A}}$ and vice versa. Celestial amplitudes are of interest for many reasons, including a holographic representation of quantum gravity in flat space $[1,2,4,17-23]$.

A central feature of the usual scattering amplitudes $\mathcal{A}$ in gravity or gauge theory is the existence of a variety of (energetically) soft theorems. These greatly constrain, and in some cases completely determine [24,25], all amplitudes. On the other hand, for celestial amplitudes there is no energy which can be taken to be soft. Instead of the energy, external particle states are characterized by their $S L(2, \mathbb{C})$ conformal dimensions $(h, \bar{h})$, which are roughly their boost weight plus or minus spin. This raises the question:

Published by the American Physical Society under the terms of the Creative Commons Attribution 4.0 International license. Further distribution of this work must maintain attribution to the author(s) and the published article's title, journal citation, and DOI. Funded by SCOAP ${ }^{3}$.
Is there an analog of the soft theorem which constrains celestial amplitudes?

The main result of this paper is to answer this question in the affirmative for non-Abelian gauge theories at tree level. The usual energetically soft theorem is replaced by the conformally soft theorem, in which the left or right conformal dimension ( $h$ or $\bar{h}$ ) of an external particle is taken to zero. The demonstration uses the energetically soft relations of $\mathcal{A}$ and is valid only when the UV behavior is sufficiently soft. Overlapping observations have been made in a number of papers including $[2,15,26]$.

From some points of view this result is expected and obvious. However, on further inspection, our derivation encounters some subtleties and relies crucially on assumed good UV behavior of the momentum-space amplitudes $\mathcal{A}$. Moreover it does not fit into our usual effective field theory worldview. The energetically soft theorem is understood to be valid because very soft photons or gluons cannot probe the shorter-distance structure of the scattering region, and can sense only the external particle trajectories which extend to infinity. Conformally soft particles, on the other hand, involve arbitrarily high energies, and their scattering might have been expected to involve UV microphysics. The fact that this is not the case cannot be understood from low-energy reasoning, but seems natural from the celestial vantage point.

The constraints on $\mathcal{A}$ imposed by the various conformally soft theorems strongly resemble those of a current algebra on a two-dimensional (2D) conformal field theory, while for example supertranslation invariance (not discussed herein) provides an infinite number of a fundamentally new type of constraints which recursively relate operators with different dimensions $[12,13,27]$. One would like to combine all known constraints and construct the most general compatible scattering amplitude. One example of an interesting question, which has been raised for example in $[24,25]$, is 
How does one characterize the most general $4 D$ fourgraviton scattering amplitude consistent with all known constraints?

The celestial picture provides a new framework for this question. Perhaps the answer is not impossibly far away.

In this paper we consider only tree-level amplitudes. Of course, the energetically soft theorem is generically corrected at the one-loop level in non-Abelian gauge theory. It would be very interesting to understand the nature of the loop corrections to the conformally soft theorem, whose form is highly constrained by the exact conformal symmetry of celestial amplitudes. See [28] for recent progress on celestial loop corrections.

This paper is organized as follows. In Sec. II we provide a general argument for the conformally soft theorem which applies to amplitudes with sufficiently soft UV behavior. This motivates a conjecture on the existence of perturbative quantum deformations of tree-level amplitudes in fourdimensional quantum field theory, which we present in Sec. III. The conformally soft theorem is explicitly verified in Sec. IV for the known tree-level celestial amplitudes, including four-point type I and heterotic string amplitudes as well as $n$-point maximally helicity violating (MHV) amplitudes in Yang-Mills theory.

\section{CONFORMALLY SOFT FROM SOFT}

In this section we derive a general identity for the scattering of conformally soft gluons in gauge theory. The main inputs are the (energetically) soft gluon theorem and assumed good UV behavior of momentum-space amplitudes.

The identity is expressed in terms of "celestial amplitudes," denoted $\tilde{\mathcal{A}}$, of gluons in a conformal primary basis given by the Mellin transform of the standard momentumspace amplitudes $\mathcal{A}[5,6]$

$\tilde{\mathcal{A}}_{J_{1} \cdots J_{n}}\left(\lambda_{i}, z_{i}, \bar{z}_{i}\right)=\left(\prod_{k=1}^{n} \int_{0}^{\infty} d \omega_{k} \omega_{k}^{i \lambda_{k}}\right) \mathcal{A}_{J_{1} \cdots J_{n}}\left(\omega_{i}, z_{i}, \bar{z}_{i}\right)$,

where $J_{i}$ label both the 4D and 2D helicities. We parametrize a null four-momentum by a sign $\epsilon_{i}= \pm 1$ (for outgoing and ingoing particles, respectively), a positive frequency $\omega_{i}$, and a point on the celestial sphere $\left(z_{i}, \bar{z}_{i}\right)$ such that

$$
p_{i}^{\mu}=\epsilon_{i} \omega_{i} q^{\mu}\left(z_{i}, \bar{z}_{i}\right)
$$

where

$$
q^{\mu}(z, \bar{z})=(1+z \bar{z}, z+\bar{z},-i(z-\bar{z}), 1-z \bar{z}) .
$$

The $\lambda_{i}$ are related to the conformal dimensions $\Delta_{i}=$ $1+i \lambda_{i}$ which in turn are related to $(h, \bar{h})$ by $(h, \bar{h})=$ $\frac{1}{2}(\Delta+J, \Delta-J)$. One may show $[5,6]$ that the celestial amplitudes have the standard $S L(2, \mathbb{C})$ Lorentz transformation property

$$
\begin{aligned}
& \tilde{\mathcal{A}}_{J_{1} \cdots J_{n}}\left(\lambda_{i}, \frac{a z_{i}+b}{c z_{i}+d}, \frac{\bar{a} \bar{z}_{i}+\bar{b}}{\bar{c} \bar{z}_{i}+\bar{d}}\right) \\
& =\prod_{j=1}^{n}\left[\left(c z_{j}+d\right)^{\Delta_{j}+J_{j}}\left(\bar{c} \bar{z}_{j}+\bar{d}\right)^{\Delta_{j}-J_{j}}\right] \tilde{\mathcal{A}}_{J_{1} \cdots J_{n}}\left(\lambda_{i}, z_{i}, \bar{z}_{i}\right) .
\end{aligned}
$$

The momentum-space amplitudes are conventionally normalized with inner product $^{1}$

$$
\left(p_{1}, J_{1} ; p_{2}, J_{2}\right)=(2 \pi)^{3} 2 p_{1}^{0} \delta^{(3)}\left(\vec{p}_{1}+\vec{p}_{2}\right) \delta_{J_{1},-J_{2}} .
$$

This implies the celestial inner product

$$
\begin{aligned}
& \left(\lambda_{1}, z_{1}, \bar{z}_{1}, J_{1} ; \lambda_{2}, z_{2}, \bar{z}_{2}, J_{2}\right) \\
& \quad=(2 \pi)^{4} \delta\left(\lambda_{1}+\lambda_{2}\right) \delta^{(2)}\left(z_{1}-z_{2}\right) \delta_{J_{1},-J_{2}} .
\end{aligned}
$$

Let us consider the $\lambda_{j} \rightarrow 0$ limit, which for a positive (negative) helicity gluon corresponds to taking $\bar{h}(h)$ to zero

$$
\begin{aligned}
\lim _{\lambda_{j} \rightarrow 0} i \lambda_{j} \tilde{\mathcal{A}}_{J_{1} \cdots J_{n}}\left(\lambda_{i}, z_{i}, \bar{z}_{i}\right) \\
=\left(\prod_{\substack{k=1 \\
k \neq j}}^{n} \int_{0}^{\infty} d \omega_{k} \omega_{k}^{i \lambda_{k}}\right)\left(\int_{0}^{\infty} d \omega_{j} \lim _{\lambda_{j} \rightarrow 0} i \lambda_{j} \omega_{j}^{i \lambda_{j}-1}\right) \\
\times \omega_{j} \mathcal{A}_{J_{1} \cdots J_{n}}\left(\omega_{i}, z_{i}, \bar{z}_{i}\right) \\
=2\left(\prod_{\substack{k=1 \\
k \neq j}}^{\infty} \int_{0}^{\infty} d \omega_{k} \omega_{k}^{i \lambda_{k}}\right) \int_{0}^{\infty} d \omega_{j} \delta\left(\omega_{j}\right) \omega_{j} \mathcal{A}_{J_{1} \cdots J_{n}}\left(\omega_{i}, z_{i}, \bar{z}_{i}\right) .
\end{aligned}
$$

Importantly, the second equality is valid only under the assumption that the momentum-space amplitudes vanish sufficiently rapidly at large momenta. This is because we have used the representation of the Dirac delta function

$$
\delta(x)=\lim _{\epsilon \rightarrow 0} \frac{\epsilon}{2}|x|^{\epsilon-1},
$$

which is only valid when integrated against functions which fall off at least as fast as $x^{-b}$ for $b>\epsilon$ as $x \rightarrow \infty .^{2}$ To study the behavior of the integrand in (2.7), we change variables $\omega_{k} \rightarrow \omega_{k} \omega_{j}$ for every $k \neq j$ yielding

\footnotetext{
${ }^{1}$ We use here the conventions of [6]. Helicities of all particles are labeled relative to the outgoing direction from the interaction.

${ }^{2}$ To see this, note the integral

$$
\int_{0}^{\infty} d x \epsilon x^{\epsilon-1} \frac{1}{(x+a)^{b}}=\frac{1}{a^{b-\epsilon}} \frac{\epsilon \Gamma(b-\epsilon) \Gamma(\epsilon)}{\Gamma(b)}, \quad a, b>0,
$$

where $b>\operatorname{Re}(\epsilon)$ in order for the integral to converge at the upper limit.
} 


$$
\begin{aligned}
\tilde{\mathcal{A}}_{J_{1} \cdots J_{n}}\left(\lambda_{i}, z_{i}, \bar{z}_{i}\right) \\
=\left(\prod_{\substack{k=1 \\
k \neq j}}^{n} \int_{0}^{\infty} d \omega_{k} \omega_{k}^{i \lambda_{k}}\right) \int_{0}^{\infty} d \omega_{j} \omega_{j}^{i \sum_{k} \lambda_{k}-1} \omega_{j}^{n} \\
\quad \times \mathcal{A}_{J_{1} \cdots J_{n}}\left(\omega_{j} \omega_{1}, z_{1}, \bar{z}_{1} ; \ldots ; \omega_{j}, z_{j}, \bar{z}_{j} ; \ldots\right) .
\end{aligned}
$$

Hence we can use the representation of the delta function (2.8) provided

$\lim _{\omega_{j} \rightarrow \infty} \omega_{j}^{n} \mathcal{A}_{J_{1} \ldots J_{n}}\left(\omega_{j} \omega_{1}, z_{1}, \bar{z}_{1} ; \ldots ; \omega_{j}, z_{j}, \bar{z}_{j} ; \ldots\right)=\mathcal{O}\left(\omega_{j}^{-\epsilon}\right)$

Tree-level pure Yang-Mills amplitudes scale homogeneously under the rescaling of all momenta

$$
\begin{gathered}
\omega_{j}^{n} \mathcal{A}_{J_{1} \cdots J_{n}}^{\text {Yang-Mills }}\left(\omega_{j} \omega_{1}, z_{1}, \bar{z}_{1} ; \ldots ; \omega_{j}, z_{j}, \bar{z}_{j} ; \ldots\right) \\
=\mathcal{A}_{J_{1} \cdots J_{n}}^{\text {Yang-Mills }}\left(\omega_{1}, z_{1}, \bar{z}_{1} ; \ldots ; 1, z_{j}, \bar{z}_{j} ; \ldots\right),
\end{gathered}
$$

and hence marginally violate (2.10). However, in string theory the tree-level four-gluon amplitudes are exponentially suppressed at large momenta [29]

$\lim _{\omega_{j} \rightarrow \infty} \omega_{j}^{4} \mathcal{A}_{J_{1} \cdots J_{4}}^{\text {string }}\left(\omega_{j} \omega_{1}, z_{1}, \bar{z}_{1} ; \ldots ; \omega_{j}, z_{j}, \bar{z}_{j} ; \ldots\right) \sim e^{-\alpha^{\prime} \omega_{j}^{2}}$,

so the use of this representation of the delta function is justified in this case.

Our approach will be to define the pure Yang-Mills celestial amplitudes as the $\alpha^{\prime} \rightarrow 0$ limit of string amplitudes. More generally various types of UV and other singularities are encountered in celestial amplitudes at both tree and loop levels. This approach potentially provides a systematic regulator for all of these singularities.

In the theory so defined, we find that the $\lambda_{j} \rightarrow 0$ limit selects the residue of the pole in the momentum-space amplitude at $\omega_{j}=0$, which is determined by the soft theorem. Using the (energetically) soft theorem for colorordered partial amplitudes

$$
\begin{aligned}
& \lim _{\omega_{j} \rightarrow 0} \omega_{j} \mathcal{A}_{J_{1} \cdots J_{n}}\left(\omega_{i}, z_{i}, \bar{z}_{i}\right) \\
& \quad=-\frac{1}{2} \frac{z_{j-1 j+1}}{z_{j-1 j} z_{j j+1}} \mathcal{A}_{J_{1} \cdots J_{j-1} J_{j+1} \cdots J_{n}}\left(\omega_{i}, z_{i}, \bar{z}_{i}\right),
\end{aligned}
$$

we obtain the tree-level conformally soft theorem

$$
\begin{aligned}
& \lim _{\lambda_{j} \rightarrow 0} i \lambda_{j} \tilde{\mathcal{A}}_{J_{1} \cdots J_{n}}\left(\lambda_{i}, z_{i}, \bar{z}_{i}\right) \\
& \quad=-\frac{1}{2} \frac{z_{j-1 j+1}}{z_{j-1 j} z_{j j+1}} \tilde{\mathcal{A}}_{J_{1} \cdots J_{j-1} J_{j+1} \cdots J_{n}}\left(\lambda_{i}, z_{i}, \bar{z}_{i}\right) .
\end{aligned}
$$

\section{A CONJECTURE}

As we have just seen, the transformation from standard scattering amplitudes (in a momentum basis) to celestial amplitudes (in a conformal basis) involves an integral over all energies. Hence, the mere existence of the celestial amplitudes requires soft UV behavior even at tree level. In Yang-Mills theory, the UV behavior is just marginally soft enough. In the scalar theories studied in $[3,7,16]$, the celestial amplitudes are also finite. In Einstein gravity, on the other hand, the Mellin transforms diverge and even the classical four-graviton celestial amplitudes do not exist, while they do appear to exist in classical string theory. It is intriguing that soft UV behavior is required even for the existence of the classical amplitudes. Of course, it is also known that there are a variety of restrictions on the UV behavior of classical scattering (such as the FroissartMartin bound) required for a unitary quantum perturbation theory. Could these be the same restrictions? This motivates the following:

CONJECTURE: A perturbative quantum deformation of a classical four-dimensional Minkowskian quantum field theory exists if and only if the celestial amplitudes $\tilde{\mathcal{A}}_{J_{1} \ldots J_{n}}\left(\lambda_{i}, z_{i}, \bar{z}_{i}\right)$ exist.

This conjecture is consistent with the (rather small) number of examples which have been analyzed so far.

\section{EXPLICIT EXAMPLES}

As a consistency check of our formal argument in Sec. II as well as to gain familiarity with celestial structures, in this section we verify that the conformally soft theorem holds for the known examples of tree-level gluon scattering in Yang-Mills and string theory.

\section{A. Review of gluon amplitudes}

In this subsection we review the expressions for celestial gluon amplitudes in both Yang-Mills and string theory $[6,9,11]$.

The general formula for $n$-point MHV Yang-Mills amplitudes in momentum space is

$$
\begin{aligned}
& \mathcal{A}_{--+\cdots+}\left(p_{1}, \ldots, p_{n}\right) \\
& =\frac{\langle 12\rangle^{3}}{\langle 23\rangle \cdots\langle n 1\rangle} \delta^{(4)}\left(\sum_{i=1}^{n} p_{i}\right) \\
& =\frac{1}{(-2)^{n-4}} \frac{z_{12}^{3}}{z_{23} \cdots z_{n 1}} \frac{\omega_{1} \omega_{2}}{\omega_{3} \cdots \omega_{n}} \delta^{(4)}\left(\sum_{i=1}^{n} \epsilon_{i} \omega_{i} q_{i}\right),
\end{aligned}
$$

where in the last equality, we used $p_{i}=\epsilon_{i} \omega_{i} q_{i}$ as in (2.2) and

$$
\langle i j\rangle=-2 \epsilon_{i} \epsilon_{j} \sqrt{\omega_{i} \omega_{j}} z_{i j} .
$$


Here and hereafter powers of the gauge (or string) coupling are absorbed into the wave function normalization. To study the three-point amplitude, we work in $(2,2)$ signature, where the amplitude is nonvanishing. $z_{i}$ and $\bar{z}_{i}$ are then independent real variables, enabling us to rewrite the momentum-conserving delta function as

$$
\begin{aligned}
\delta^{(4)} & \left(\sum_{i=1}^{3} \epsilon_{i} \omega_{i} q_{i}\right) \\
= & \frac{\operatorname{sgn}\left(z_{23} z_{31}\right)}{4 \omega_{3}^{2} z_{23} z_{31}} \delta\left(\omega_{1}-\frac{\omega_{3} \epsilon_{3}}{\epsilon_{1}} \frac{z_{23}}{z_{12}}\right) \delta\left(\omega_{2}-\frac{\omega_{3} \epsilon_{3}}{\epsilon_{2}} \frac{z_{31}}{z_{12}}\right) \\
& \times \delta\left(\bar{z}_{13}\right) \delta\left(\bar{z}_{23}\right),
\end{aligned}
$$

where the form above assumes $z_{i j} \neq 0$. An alternate form may be found by instead assuming $\bar{z}_{i j} \neq 0$,

$$
\begin{aligned}
\delta^{(4)} & \left(\sum_{i=1}^{3} \epsilon_{i} \omega_{i} q_{i}\right) \\
= & \frac{\operatorname{sgn}\left(\bar{z}_{23} \bar{z}_{31}\right)}{4 \omega_{3}^{2} \bar{z}_{23} \bar{z}_{31}} \delta\left(\omega_{1}-\frac{\omega_{3} \epsilon_{3}}{\epsilon_{1}} \frac{\bar{z}_{23}}{\bar{z}_{12}}\right) \delta\left(\omega_{2}-\frac{\omega_{3} \epsilon_{3}}{\epsilon_{2}} \frac{\bar{z}_{31}}{\bar{z}_{12}}\right) \\
& \times \delta\left(z_{13}\right) \delta\left(z_{23}\right) .
\end{aligned}
$$

For --+ scattering we use (4.3) in which case the resulting celestial amplitude is

$$
\begin{aligned}
\tilde{\mathcal{A}}_{--+} & \left(\lambda_{i}, z_{i}, \bar{z}_{i}\right) \\
= & -\pi \delta\left(\lambda_{1}+\lambda_{2}+\lambda_{3}\right) \frac{z_{12}^{3}}{z_{23}^{2} z_{31}^{2}}\left(\frac{\epsilon_{3}}{\epsilon_{1}} \frac{z_{23}}{z_{12}}\right)^{1+i \lambda_{1}}\left(\frac{\epsilon_{3}}{\epsilon_{2}} \frac{z_{31}}{z_{12}}\right)^{1+i \lambda_{2}} \\
& \times \operatorname{sgn}\left(z_{23} z_{31}\right) \delta\left(\bar{z}_{13}\right) \delta\left(\bar{z}_{23}\right) \Theta\left(\frac{\epsilon_{3}}{\epsilon_{1}} \frac{z_{23}}{z_{12}}\right) \Theta\left(\frac{\epsilon_{3}}{\epsilon_{2}} \frac{z_{31}}{z_{12}}\right) .
\end{aligned}
$$

Here $\Theta$ vanishes (equals unity) for negative (positive) argument.

Similarly, to determine the celestial four-point amplitude, the trick is to rewrite the momentum-conserving delta function

$$
\begin{aligned}
\delta^{(4)} & \left(\sum_{i=1}^{4} \epsilon_{i} \omega_{i} q_{i}\right) \\
= & \frac{1}{4 \omega_{3}} \delta\left(\omega_{1}-\frac{\omega_{3} \epsilon_{3}}{\epsilon_{1}} \frac{z_{23} \bar{z}_{34}}{z_{12} \bar{z}_{14}}\right) \delta\left(\omega_{2}-\frac{\omega_{3} \epsilon_{3}}{\epsilon_{2}} \frac{z_{13} \bar{z}_{34}}{z_{12} \bar{z}_{42}}\right) \\
& \times \delta\left(\omega_{4}-\frac{\omega_{3} \epsilon_{3}}{\epsilon_{4}} \frac{\bar{z}_{13} z_{23}}{\bar{z}_{14} z_{42}}\right) \delta\left(z_{12} z_{34} \bar{z}_{13} \bar{z}_{24}-z_{13} z_{24} \bar{z}_{12} \bar{z}_{34}\right),
\end{aligned}
$$

from which it directly follows that

$$
\begin{aligned}
\tilde{\mathcal{A}}_{--++}\left(\lambda_{i}, z_{i}, \bar{z}_{i}\right) \\
=\frac{\pi}{2} \delta\left(\sum_{i=1}^{4} \lambda_{i}\right) \frac{z_{12}^{3}}{z_{23} z_{34} z_{41}}\left(\frac{\epsilon_{3}}{\epsilon_{1}} \frac{z_{23} \bar{z}_{34}}{z_{12} \bar{z}_{14}}\right)^{1+i \lambda_{1}}\left(\frac{\epsilon_{3}}{\epsilon_{2}} \frac{z_{13} \bar{z}_{34}}{z_{12} \bar{z}_{42}}\right)^{1+i \lambda_{2}} \\
\quad \times\left(\frac{\epsilon_{3}}{\epsilon_{4}} \frac{\bar{z}_{13} z_{23}}{\bar{z}_{14} z_{42}}\right)^{-1+i \lambda_{4}} \delta\left(z_{12} z_{34} \bar{z}_{13} \bar{z}_{24}-z_{13} z_{24} \bar{z}_{12} \bar{z}_{34}\right) \\
\quad \times \Theta\left(\frac{\epsilon_{3}}{\epsilon_{1}} \frac{z_{23} \bar{z}_{34}}{z_{12} \bar{z}_{14}}\right) \Theta\left(\frac{\epsilon_{3}}{\epsilon_{2}} \frac{z_{13} \bar{z}_{34}}{z_{12} \bar{z}_{42}}\right) \Theta\left(\frac{\epsilon_{3}}{\epsilon_{4}} \frac{\bar{z}_{13} z_{23}}{\bar{z}_{14} z_{42}}\right) .
\end{aligned}
$$

The factor of $\delta\left(\sum \lambda_{i}\right)$ arises from the scale invariance of Yang-Mills amplitudes in momentum space and generalizes to the $n$-point amplitude.

In momentum space, the four-gluon amplitudes in type I and heterotic string theory differ from the Yang-Mills amplitude by a multiplicative factor $[30,31]$

$$
\begin{aligned}
\mathcal{A}_{--++}^{\text {type I }} & =\frac{\Gamma(1-s) \Gamma(1-u)}{\Gamma(1-s-u)} \mathcal{A}_{--++}^{\text {Yang-Mills }}, \\
\mathcal{A}_{--++}^{\text {heterotic }} & =-\frac{\Gamma(-s) \Gamma(-t) \Gamma(-u)}{\Gamma(s) \Gamma(t) \Gamma(u)} \mathcal{A}_{--++}^{\text {Yang-Mills }},
\end{aligned}
$$

where $s=p_{12}, u=p_{23}, t=p_{13}$ with

$$
p_{i j}=-\alpha^{\prime}\left(p_{i}+p_{j}\right)^{2}=4 \alpha^{\prime} \epsilon_{i} \epsilon_{j} \omega_{i} \omega_{j} z_{i j} \bar{z}_{i j} .
$$

As string amplitudes are not scale invariant, the $\delta\left(\sum \lambda_{i}\right)$ factor no longer appears. As shown in [11], the celestial four-gluon amplitudes in type I and heterotic string theory are obtained by the replacements

$$
\begin{aligned}
(2 \pi) \delta\left(\sum_{i=1}^{4} \lambda_{i}\right) & \rightarrow \int_{0}^{\infty} d \omega \omega^{i\left(\lambda_{1}+\lambda_{2}+\lambda_{3}+\lambda_{4}\right)-1} \\
& \times\left(\frac{\Gamma(1-s(\omega)) \Gamma(1-u(\omega))}{\Gamma(1-s(\omega)-u(\omega))}\right) \\
& \equiv F_{I}
\end{aligned}
$$

and

$$
\begin{aligned}
(2 \pi) \delta\left(\sum_{i=1}^{4} \lambda_{i}\right) \rightarrow & \int_{0}^{\infty} d \omega \omega^{i\left(\lambda_{1}+\lambda_{2}+\lambda_{3}+\lambda_{4}\right)-1} \\
& \times\left(-\frac{\Gamma(-s(\omega)) \Gamma(-t(\omega)) \Gamma(-u(\omega))}{\Gamma(s(\omega)) \Gamma(t(\omega)) \Gamma(u(\omega))}\right) \\
\equiv & F_{H}
\end{aligned}
$$

in (4.7), respectively. Here 


$$
\begin{aligned}
& s(\omega)=4 \alpha^{\prime} \omega^{2} \frac{z_{23} \bar{z}_{13}}{z_{42} \bar{z}_{14}} z_{34} \bar{z}_{34}, \quad u(\omega)=4 \alpha^{\prime} \omega^{2} \frac{z_{13} \bar{z}_{34}}{z_{12} \bar{z}_{42}} z_{23} \bar{z}_{23}, \\
& t(\omega)=4 \alpha^{\prime} \omega^{2} \frac{z_{23} \bar{z}_{34}}{z_{12} \bar{z}_{14}} z_{13} \bar{z}_{13} .
\end{aligned}
$$

A salient feature of celestial string amplitudes with five or more gluons is - assuming soft high-energy behaviorthat they appear to be finite at generic values of $\left(\lambda_{i}, z_{i}\right)$. This contrasts with the momentum-space amplitudes, which vanish when momentum is not conserved and are infinite when it is (due to the delta function). For four gluons, however, all celestial amplitudes develop a kinematic delta-function singularity arising from translation invariance, which restricts the $2 \mathrm{D}$ conformal cross ratio to lie on the real axis. This can be seen directly from (4.6). For three gluons further kinematic singularities appear in $(2,2)$ signature, while in $(3,1)$ the amplitudes vanish. In the $\alpha^{\prime} \rightarrow 0$ Yang-Mills limit, the celestial amplitudes are no longer generically finite due to the appearance of the $\delta\left(\sum \lambda_{i}\right)$ factor. While the regulation and properties of momentumspace delta-function singularities are long understood, the delta-function singularities of celestial amplitudes are rather different and remain to be fully understood.

\section{B. Four-point Yang-Mills amplitude}

In this section we explicitly study the conformally soft limit of the four-point Yang-Mills gluon amplitude (4.7). Taking $\lambda_{4} \rightarrow 0$ and using the identity (2.8) introduces an additional delta function on the right-hand side of (4.7),

$$
\begin{aligned}
& \lim _{\lambda_{4} \rightarrow 0} i \lambda_{4} \tilde{\mathcal{A}}_{--++}\left(\lambda_{i}, z_{i}, \bar{z}_{i}\right) \\
& =\delta\left(\frac{\epsilon_{3}}{\epsilon_{4}} \frac{\bar{z}_{13} z_{23}}{\bar{z}_{14} z_{42}}\right)\left[\pi \delta\left(\sum_{i=1}^{3} \lambda_{i}\right) \frac{z_{12}^{3}}{z_{23} z_{34} z_{41}}\left(\frac{\epsilon_{3}}{\epsilon_{1}} \frac{z_{23} \bar{z}_{34}}{z_{12} \bar{z}_{14}}\right)^{1+i \lambda_{1}}\right. \\
& \times\left(\frac{\epsilon_{3}}{\epsilon_{2}} \frac{z_{13} \bar{z}_{34}}{z_{12} \bar{z}_{42}}\right)^{1+i \lambda_{2}} \delta\left(z_{12} z_{34} \bar{z}_{13} \bar{z}_{24}-z_{13} z_{24} \bar{z}_{12} \bar{z}_{34}\right) \\
& \left.\times \Theta\left(\frac{\epsilon_{3}}{\epsilon_{1}} \frac{z_{23} \bar{z}_{34}}{z_{12} \bar{z}_{14}}\right) \Theta\left(\frac{\epsilon_{3}}{\epsilon_{2}} \frac{z_{13} \bar{z}_{34}}{z_{12} \bar{z}_{42}}\right) \Theta\left(\frac{\epsilon_{3}}{\epsilon_{4}} \frac{\bar{z}_{13} z_{23}}{\bar{z}_{14} z_{42}}\right)\right] \text {. }
\end{aligned}
$$

Assuming

$$
z_{23} \neq 0
$$

where we are taking $z_{i}$ and $\bar{z}_{i}$ to be independent real variables, the additional delta function can be written as

$$
\delta\left(\frac{\epsilon_{3}}{\epsilon_{4}} \frac{\bar{z}_{13} z_{23}}{\bar{z}_{14} z_{42}}\right)=\operatorname{sgn}\left(\bar{z}_{14} z_{42} z_{23}\right) \frac{\bar{z}_{14} z_{42}}{z_{23}} \delta\left(\bar{z}_{13}\right) .
$$

Then, Eq. (4.13) simplifies to

$$
\begin{aligned}
\lim _{\lambda_{4} \rightarrow 0} i \lambda_{4} & \tilde{\mathcal{A}}_{--++}\left(\lambda_{i}, z_{i}, \bar{z}_{i}\right) \\
= & \operatorname{sgn}\left(\bar{z}_{14} z_{42} z_{23}\right) \frac{\bar{z}_{14} z_{42}}{z_{23}} \delta\left(\bar{z}_{13}\right) \\
& \times\left[\frac{1}{4}(2 \pi) \delta\left(\sum_{i=1}^{3} \lambda_{i}\right) \frac{z_{12}^{3}}{z_{23} z_{34} z_{41}}\left(\frac{\epsilon_{3}}{\epsilon_{1}} \frac{z_{23}}{z_{12}}\right)^{1+i \lambda_{1}}\right. \\
& \times\left(\frac{\epsilon_{3}}{\epsilon_{2}} \frac{z_{13} \bar{z}_{34}}{z_{12} \bar{z}_{42}}\right)^{1+i \lambda_{2}} \delta\left(z_{13} z_{24} \bar{z}_{12} \bar{z}_{34}\right) \\
& \left.\times \Theta\left(\frac{\epsilon_{3}}{\epsilon_{1}} \frac{z_{23}}{z_{12}}\right) \Theta\left(\frac{\epsilon_{3}}{\epsilon_{2}} \frac{z_{13} \bar{z}_{34}}{z_{12} \bar{z}_{42}}\right)\right]
\end{aligned}
$$

where we find the argument of one of the step functions vanishes and we take $\Theta(0)=\frac{1}{2}$.

To further simplify, we focus on the other delta function by assuming ${ }^{3}$

$$
z_{13} \neq 0, \quad z_{24} \neq 0, \quad \text { and } \quad \bar{z}_{34} \neq 0,
$$

so that

$$
\delta\left(z_{13} z_{24} \bar{z}_{12} \bar{z}_{34}\right)=\frac{\operatorname{sgn}\left(z_{13} z_{24} \bar{z}_{34}\right)}{z_{13} z_{24} \bar{z}_{34}} \delta\left(\bar{z}_{12}\right) .
$$

Using this, the amplitude (4.16) further simplifies as

$$
\begin{aligned}
& \lim _{\lambda_{4} \rightarrow 0} i \lambda_{4} \tilde{\mathcal{A}}_{--++}\left(\lambda_{i}, z_{i}, \bar{z}_{i}\right) \\
& =\frac{\operatorname{sgn}\left(z_{23} z_{31}\right)}{z_{31} z_{23}} \delta\left(\bar{z}_{12}\right) \delta\left(\bar{z}_{13}\right) \\
& \quad \times\left[\frac{1}{4}(2 \pi) \delta\left(\sum_{i=1}^{3} \lambda_{i}\right) \frac{z_{12}^{3}}{z_{23} z_{34} z_{41}}\left(\frac{\epsilon_{3}}{\epsilon_{1}} \frac{z_{23}}{z_{12}}\right)^{1+i \lambda_{1}}\left(\frac{\epsilon_{3}}{\epsilon_{2}} \frac{z_{31}}{z_{12}}\right)^{1+i \lambda_{2}}\right. \\
& \left.\quad \times \Theta\left(\frac{\epsilon_{3}}{\epsilon_{1}} \frac{z_{23}}{z_{12}}\right) \Theta\left(\frac{\epsilon_{3}}{\epsilon_{2}} \frac{z_{31}}{z_{12}}\right)\right] .
\end{aligned}
$$

Comparing with our expression for the three-point celestial amplitude (4.5), we verify the anticipated relation (2.14)

$$
\lim _{\lambda_{4} \rightarrow 0} i \lambda_{4} \tilde{\mathcal{A}}_{--++}\left(\lambda_{i}, z_{i}, \bar{z}_{i}\right)=-\frac{1}{2} \frac{z_{31}}{z_{34} z_{41}} \tilde{\mathcal{A}}_{--+}\left(\lambda_{i}, z_{i}, \bar{z}_{i}\right) .
$$

\section{Four-point type I amplitude}

In this subsection we study the conformally soft limit of the four-point type I gluon amplitude

\footnotetext{
${ }^{3}$ We make such assumptions here because in this section we are interested in the conformally soft singularity structure of the amplitude as opposed to the collinear singularity structure. We do not study the regime in which the singularities overlap.
} 


$$
\begin{aligned}
& \tilde{\mathcal{A}}_{--++}^{\text {type I }}\left(\lambda_{i}, z_{i}, \bar{z}_{i}\right) \\
& =\frac{1}{4} F_{I} \frac{z_{12}^{3}}{z_{23} z_{34} z_{41}}\left(\frac{\epsilon_{3}}{\epsilon_{1}} \frac{z_{23} \bar{z}_{34}}{z_{12} \bar{z}_{14}}\right)^{1+i \lambda_{1}}\left(\frac{\epsilon_{3}}{\epsilon_{2}} \frac{z_{13} \bar{z}_{34}}{z_{12} \bar{z}_{42}}\right)^{1+i \lambda_{2}} \\
& \times\left(\frac{\epsilon_{3}}{\epsilon_{4}} \frac{\bar{z}_{13} z_{23}}{\bar{z}_{14} z_{42}}\right)^{-1+i \lambda_{4}} \delta\left(z_{12} z_{34} \bar{z}_{13} \bar{z}_{24}-z_{13} z_{24} \bar{z}_{12} \bar{z}_{34}\right) \\
& \times \Theta\left(\frac{\epsilon_{3}}{\epsilon_{1}} \frac{z_{23} \bar{z}_{34}}{z_{12} \bar{z}_{14}}\right) \Theta\left(\frac{\epsilon_{3}}{\epsilon_{2}} \frac{z_{13} \bar{z}_{34}}{z_{12} \bar{z}_{42}}\right) \Theta\left(\frac{\epsilon_{3}}{\epsilon_{4}} \frac{\bar{z}_{13} z_{23}}{\bar{z}_{14} z_{42}}\right),
\end{aligned}
$$

with $F_{I}$ given in (4.10). Performing the same limit as in pure Yang-Mills (4.13), again we obtain an additional delta function on the right-hand side of (4.21)

$$
\begin{aligned}
& \lim _{\lambda_{4} \rightarrow 0} i \lambda_{4} \tilde{\mathcal{A}}_{--++}^{\text {type I }}\left(\lambda_{i}, z_{i}, \bar{z}_{i}\right) \\
& =\delta\left(\frac{\epsilon_{3}}{\epsilon_{4}} \frac{\bar{z}_{13} z_{23}}{\bar{z}_{14} z_{42}}\right)\left[\frac{1}{2}\left(\left.F_{I}\right|_{\lambda_{4}=0}\right) \frac{z_{12}^{3}}{z_{23} z_{34} z_{41}}\left(\frac{\epsilon_{3}}{\epsilon_{1}} \frac{z_{23} \bar{z}_{34}}{z_{12} \bar{z}_{14}}\right)^{1+i \lambda_{1}}\right. \\
& \times\left(\frac{\epsilon_{3}}{\epsilon_{2}} \frac{z_{13} \bar{z}_{34}}{z_{12} \bar{z}_{42}}\right)^{1+i \lambda_{2}} \delta\left(z_{12} z_{34} \bar{z}_{13} \bar{z}_{24}-z_{13} z_{24} \bar{z}_{12} \bar{z}_{34}\right) \\
& \left.\times \Theta\left(\frac{\epsilon_{3}}{\epsilon_{1}} \frac{z_{23} \bar{z}_{34}}{z_{12} \bar{z}_{14}}\right) \Theta\left(\frac{\epsilon_{3}}{\epsilon_{2}} \frac{z_{13} \bar{z}_{34}}{z_{12} \bar{z}_{42}}\right) \Theta\left(\frac{\epsilon_{3}}{\epsilon_{4}} \frac{\bar{z}_{13} z_{23}}{\bar{z}_{14} z_{42}}\right)\right] \text {. }
\end{aligned}
$$

Assuming as before that $z_{23} \neq 0$ so (4.15) holds, we find the right-hand side of (4.22) reduces to the same expression as in pure Yang-Mills (4.16), since from (4.10) and (4.12) one readily finds

$$
\begin{aligned}
\left.F_{I}\right|_{\substack{\lambda_{4}=0 \\
\bar{z}_{13}=0}} & =\int_{0}^{\infty} d \omega \omega^{i\left(\lambda_{1}+\lambda_{2}+\lambda_{3}\right)-1} \\
& =2 \pi \delta\left(\lambda_{1}+\lambda_{2}+\lambda_{3}\right) .
\end{aligned}
$$

Then, by following the remaining steps that were applied to the pure Yang-Mills amplitude, one concludes

$$
\lim _{\lambda_{4} \rightarrow 0} i \lambda_{4} \tilde{\mathcal{A}}_{--++}^{\mathrm{typeI}}\left(\lambda_{i}, z_{i}, \bar{z}_{i}\right)=-\frac{1}{2} \frac{z_{31}}{z_{34} z_{41}} \tilde{\mathcal{A}}_{--+}\left(\lambda_{i}, z_{i}, \bar{z}_{i}\right) .
$$

The amplitude appearing on the right-hand side is the three-point celestial amplitude (4.5), which is identical in Yang-Mills and type I string theory.

\section{Four-point heterotic amplitude}

From the previous section, we learned that in order to show that the four-point amplitude in heterotic string theory obeys the same Ward identity as the four-point type I and Yang-Mills amplitudes, we first need to determine

$$
\left.F_{H}\right|_{\substack{\lambda_{4}=0 \\ \bar{z}_{13}=0}}=\int_{0}^{\infty} d \omega \omega^{i\left(\lambda_{1}+\lambda_{2}+\lambda_{3}\right)-1}\left(-\frac{\Gamma(-u(\omega))}{\Gamma(u(\omega))}\right) .
$$

This readily follows from Eqs. (4.11) and (4.12) and the fact

$$
\lim _{x \rightarrow 0} \frac{\Gamma(x)}{\Gamma(-x)}=-1
$$

Using this and following the steps outlined in the previous section, we arrive at

$$
\begin{aligned}
\lim _{\lambda_{4} \rightarrow 0} i \lambda_{4} \tilde{\mathcal{A}}_{--++}^{\text {heterotic }}\left(\lambda_{i}, z_{i}, \bar{z}_{i}\right) \\
=\operatorname{sgn}\left(\bar{z}_{14} z_{42} z_{23}\right) \frac{\bar{z}_{14} z_{42}}{z_{23}} \delta\left(\bar{z}_{13}\right)\left[\frac { 1 } { 2 } \left(F_{H} \mid \begin{array}{l}
\lambda_{4}=0 \\
\bar{z}_{13}=0 \\
z_{23} z_{34} z_{41}
\end{array}\right.\right. \\
\quad \times\left(\frac{\epsilon_{3}}{\epsilon_{1}} \frac{z_{23}^{3}}{z_{12}}\right)^{1+i \lambda_{1}}\left(\frac{\epsilon_{3}}{\epsilon_{2}} \frac{z_{13} \bar{z}_{34}}{z_{12} \bar{z}_{42}}\right)^{1+i \lambda_{2}} \delta\left(z_{13} z_{24} \bar{z}_{12} \bar{z}_{34}\right) \\
\left.\quad \times \Theta\left(\frac{\epsilon_{3}}{\epsilon_{1}} \frac{z_{23}}{z_{12}}\right) \Theta\left(\frac{\epsilon_{3}}{\epsilon_{2}} \frac{z_{13} \bar{z}_{34}}{z_{12} \bar{z}_{42}}\right) \Theta(0)\right] .
\end{aligned}
$$

Assuming a noncollinear configuration such that (4.18) applies, the right-hand side of (4.27) reduces to the expression for pure Yang-Mills (4.19) since

$$
\left.F_{H}\right|_{\substack{\lambda_{4}=0 \\ z_{13}=0 \\ z_{12}=0}}=(2 \pi) \delta\left(\lambda_{1}+\lambda_{2}+\lambda_{3}\right)
$$

Thus, the heterotic four-gluon amplitude likewise obeys the Ward identity

$$
\lim _{\lambda_{4} \rightarrow 0} i \lambda_{4} \tilde{\mathcal{A}}_{--++}^{\text {heterotic }}\left(\lambda_{i}, z_{i}, \bar{z}_{i}\right)=-\frac{1}{2} \frac{z_{31}}{z_{34} z_{41}} \tilde{\mathcal{A}}_{--+}\left(\lambda_{i}, z_{i}, \bar{z}_{i}\right) .
$$

\section{E. Permuting external legs}

In momentum space, the four-point MHV amplitude is symmetric under the exchange of 3 and 4 , and consequently the soft limits $\omega_{3}$ and $\omega_{4}$ can both be readily obtained from the momentum-space expression (4.1). On the other hand, the four-point celestial amplitudes were written in Sec. IVA in a form that elucidates their behavior in the $\lambda_{4} \rightarrow 0$ conformally soft limit, but obscures their behavior in the $\lambda_{3} \rightarrow 0$ conformally soft limit. Of course, one can start from the momentum-space amplitudes and derive a different expression for the celestial amplitudes in which the $\lambda_{3} \rightarrow 0$ conformally soft limit can easily be taken. However, one of the goals of this paper is to understand everything directly in the conformal basis, so in this subsection we explain how to permute 3 and 4 without reference to the momentum-space representation. We shall see the permutation symmetry is related to scaling properties of the amplitudes. The general form of the four-gluon celestial amplitude is 


$$
\begin{aligned}
& \tilde{\mathcal{A}}_{--++}\left(\lambda_{i}, z_{i}, \bar{z}_{i}\right) \\
& =\frac{1}{4} F\left(\lambda_{i}, \hat{s}, \hat{u}\right)\left(\frac{\epsilon_{3} z_{23} \bar{z}_{34}}{\epsilon_{1} z_{12} \bar{z}_{14}}\right)^{1+i \lambda_{1}}\left(\frac{\epsilon_{3} z_{13} \bar{z}_{34}}{\epsilon_{2} z_{12} \bar{z}_{42}}\right)^{1+i \lambda_{2}} \\
& \times\left(\frac{\epsilon_{3} \bar{z}_{13} z_{23}}{\epsilon_{4}} \bar{z}_{14} z_{42}\right)^{-1+i \lambda_{4}} \frac{z_{12}^{3}}{z_{23} z_{34} z_{41}} \delta\left(z_{12} z_{34} \bar{z}_{13} \bar{z}_{24}-z_{13} z_{24} \bar{z}_{12} \bar{z}_{34}\right) \\
& \times \Theta\left(\frac{\epsilon_{3}}{\epsilon_{1}} \frac{z_{23} \bar{z}_{34}}{z_{12} \bar{z}_{14}}\right) \Theta\left(\frac{\epsilon_{3}}{\epsilon_{2}} \frac{z_{13} \bar{z}_{34}}{z_{12} \bar{z}_{42}}\right) \Theta\left(\frac{\epsilon_{3}}{\epsilon_{4}} \frac{\bar{z}_{13} z_{23}}{\bar{z}_{14} z_{42}}\right),
\end{aligned}
$$

where

$$
F\left(\lambda_{i}, \hat{s}, \hat{u}\right)=\int_{0}^{\infty} d \omega \omega^{-1+i} \sum_{i=1}^{4} \lambda_{i} f\left(\omega^{2} \hat{s}, \omega^{2} \hat{u}\right),
$$

$\omega^{2} \hat{s}=s$, and $\omega^{2} \hat{u}=u$, with $s$ and $u$ given in (4.12) and related to $t$ by $s+t+u=0$.

Now suppose we perform a change of variables $\omega \rightarrow \omega \alpha$, where $\alpha>0$. We find

$$
F\left(\lambda_{i}, \hat{s}, \hat{u}\right)=\alpha^{i \sum_{i=1}^{4} \lambda_{i}} F\left(\lambda_{i}, \alpha^{2} \hat{s}, \alpha^{2} \hat{u}\right),
$$

and in the full amplitude,

$$
\begin{aligned}
\tilde{\mathcal{A}}_{--++}\left(\lambda_{i}, z_{i}, \bar{z}_{i}\right) \\
=\frac{1}{4} F\left(\lambda_{i}, \alpha^{2} \hat{s}, \alpha 2 \hat{u}\right)\left(\alpha \frac{\epsilon_{3}}{\epsilon_{1}} \frac{z_{23} \bar{z}_{34}}{z_{12} \bar{z}_{14}}\right)^{1+i \lambda_{1}} \\
\quad \times\left(\alpha \frac{\epsilon_{3}}{\epsilon_{2}} \frac{z_{13} \bar{z}_{34}}{z_{12} \bar{z}_{42}}\right)^{1+i \lambda_{2}}\left(\alpha \frac{\epsilon_{3}}{\epsilon_{4}} \frac{\bar{z}_{13} z_{23}}{\bar{z}_{14} z_{42}}\right)^{-1+i \lambda_{4}} \alpha^{-1+i \lambda_{3}} \\
\quad \times \frac{z_{12}^{3}}{z_{23} z_{34} z_{41}} \delta\left(z_{12} z_{34} \bar{z}_{13} \bar{z}_{24}-z_{13} z_{24} \bar{z}_{12} \bar{z}_{34}\right) \\
\quad \times \Theta\left(\frac{\epsilon_{3}}{\epsilon_{1}} \frac{z_{23} \bar{z}_{34}}{z_{12} \bar{z}_{14}}\right) \Theta\left(\frac{\epsilon_{3}}{\epsilon_{2}} \frac{z_{13} \bar{z}_{34}}{z_{12} \bar{z}_{42}}\right) \Theta\left(\frac{\epsilon_{3}}{\epsilon_{4}} \frac{\bar{z}_{13} z_{23}}{\bar{z}_{14} z_{42}}\right) .
\end{aligned}
$$

Then, if we choose

$$
\alpha=\left(\frac{\epsilon_{3}}{\epsilon_{4}} \frac{\bar{z}_{13} z_{23}}{\bar{z}_{14} z_{42}}\right)^{-1}
$$

which is guaranteed to be positive by one of the step functions, we obtain a form of the amplitude in which we can easily study the limit $\lambda_{3} \rightarrow 0$.

\section{F. $\boldsymbol{n}$-point MHV Yang-Mills amplitude}

The $n$-point MHV gluon amplitudes were derived in terms of generalized hypergeometric functions by Schreiber, Volovich, and Zlotnikov [9]. In the Appendix we verify that these amplitudes are related by the conformally soft relation (2.14). In this section, we present an alternate route to the same end. We first present a new formula for the $n$-point MHV celestial amplitude which manifestly exhibits all poles in $\lambda_{i}$ for $i=3, \ldots, n$, allowing a streamlined analysis of the conformally soft limit. Moreover, for $n>4$, the absence of poles in $\lambda_{1}, \lambda_{2}$ implies $\lim _{\lambda_{i} \rightarrow 0} \lambda_{i} \tilde{\mathcal{A}}_{--+\ldots+}^{(n)}\left(\lambda_{i}, z_{i}, \bar{z}_{i}\right)=0$ for $i=1$, 2, which is consistent with the vanishing of the momentum-space soft limit in these cases due to $\mathcal{A}_{-+\cdots+}^{(n-1)}\left(\omega_{i}, z_{i}, \bar{z}_{i}\right)=0$. In the $n=4$ case, the conformally soft limit of the negative helicity gluons in (4.7) yields the expected conformally soft formula.

The Mellin transform of the $n$-point MHV amplitude can be written

$$
\begin{aligned}
\tilde{\mathcal{A}}_{--+\cdots+}^{(n)}\left(\lambda_{i}, z_{i}, \bar{z}_{i}\right) & =\frac{1}{(-2)^{n-4}} \frac{z_{12}^{3}}{z_{23} \cdots z_{n 1}} \prod_{i=1}^{n}\left(\int_{0}^{\infty} d \omega_{i} \omega_{i}^{i \lambda_{i}}\right) \frac{\omega_{1} \omega_{2}}{\omega_{3} \cdots \omega_{n}} \delta^{(4)}\left(\sum_{i=1}^{n} \epsilon_{i} \omega_{i} q_{i}\right) \\
& =\frac{1}{(-2)^{n-4}} \frac{z_{12}^{3}}{z_{23} \cdots z_{n 1}} \prod_{i=1}^{n}\left(\int_{0}^{\infty} d \omega_{i} \omega_{i}^{i \lambda_{i}-J_{i}}\right) \int \frac{d^{4} y}{(2 \pi)^{4}} e^{-i \sum \omega_{i}\left(\epsilon_{i} y \cdot q_{i}-i \varepsilon\right)} \\
& =\frac{1}{(-2)^{n-4}} \frac{1}{(2 \pi)^{4}} \frac{z_{12}^{3}}{z_{23} \cdots z_{n 1}} \int d^{4} y \prod_{i=1}^{n} \frac{\Gamma\left(i \lambda_{i}+1-J_{i}\right)}{\left[i \epsilon_{i} y \cdot q_{i}+\varepsilon\right]^{i \lambda_{i}+1-J_{i}}} .
\end{aligned}
$$

In the last line we used the formula

$$
\int_{0}^{\infty} e^{-a \omega} \omega^{z} d \omega=\frac{\Gamma(z+1)}{a^{z+1}}
$$

We see explicitly from the $\Gamma$ functions that $\tilde{\mathcal{A}}_{--+\cdots+}^{(n)}\left(\lambda_{i}, z_{i}, \bar{z}_{i}\right)$ has poles in $\lambda_{i}$ for $3 \leq i \leq n$ (but not in $\lambda_{1}$ or $\lambda_{2}$ ) so the conformally soft limit picks out the residue of the pole. Since the factor $z_{12}^{3} /\left(z_{23} \cdots z_{n 1}\right)$ supplies the appropriate soft factor $z_{i-1 i+1} /\left(z_{i-1 i} z_{i i+1}\right)$, the amplitude obeys the conformally soft theorem so long as the remaining part becomes independent of $\left(z_{i}, \bar{z}_{i}\right)$ in the limit $\lambda_{i} \rightarrow 0$. Indeed, in this limit, the factor associated with the $i$ th particle $\left[i \epsilon_{i} y \cdot q_{i}+\varepsilon\right]^{-i \lambda_{i}-1+J_{i}} \rightarrow 1$, so the conformally soft formula (2.14) holds.

\section{ACKNOWLEDGMENTS}

We are grateful to Nima Arkani-Hamed, Laura Donnay, Charles Marteau, Andrea Puhm, Anders Schreiber, Marcus Spradlin, Tomasz Taylor, and Ellis Yuan for useful 
discussions. This work was supported by DOE Grant No. de-sc0007870.

\section{APPENDIX: SCHREIBER-VOLOVICH- ZLOTNIKOV MHV AMPLITUDES}

In this Appendix we analyze the soft limits of MHV amplitudes using the representation of the $n$-point celestial amplitude in terms of generalized hypergeometric functions given by Schreiber, Volovich, and Zlotnikov [9]. They found $^{4}$

$$
\begin{aligned}
\tilde{\mathcal{A}}_{--+\cdots+}^{(n)}\left(\lambda_{i}, z_{i}, \bar{z}_{i}\right) \\
=\frac{2 \pi}{(-2)^{n-4}} \frac{z_{12}^{3}}{z_{23} \cdots z_{n 1}} \frac{1}{\left|U_{1, n}\right|} \\
\quad \times \delta\left(\sum_{j=1}^{n} \lambda_{j}\right) \hat{\varphi}_{n}(\{\alpha\}, x) \prod_{i, j} \Theta\left(-\frac{U_{i, j}}{U}\right),
\end{aligned}
$$

where $i=1, \ldots, n-4, j=n-3, \ldots, n$, and

$$
\begin{aligned}
& \hat{\varphi}_{n}(\{\alpha\}, x) \\
= & \frac{c_{2}}{c_{1}} \int_{u_{1}, \ldots, u_{3}, 1-\sum_{a=1}^{3} u_{a} \geq 0} \prod_{j=1}^{n} P_{j}^{\alpha_{j}} \frac{d P_{n-3} \wedge d P_{n-2} \wedge d P_{n-1}}{P_{n-3} P_{n-2} P_{n-1}}, \\
P_{j} & =x_{0 j}+x_{1 j} u_{1}+x_{2 j} u_{2}+x_{3 j} u_{3}, \\
\frac{c_{2}}{c_{1}} & =\frac{\Gamma\left(2+i \lambda_{1}\right) \Gamma\left(2+i \lambda_{2}\right) \prod_{j=3}^{n-4} \Gamma\left(i \lambda_{j}\right)}{\Gamma\left(1-i \lambda_{1}\right) \prod_{i=1}^{3} \Gamma\left(1-i \lambda_{n-i}\right)}
\end{aligned}
$$

are dual integral representations of hypergeometric functions. The parameters $\alpha_{i}$ and $x_{i j}$ are given by

$$
\begin{aligned}
\alpha_{1} & =1, \quad \alpha_{2}=-2-i \lambda_{2}, \quad \alpha_{3}=-i \lambda_{3}, \ldots, \\
\alpha_{n-4} & =-i \lambda_{n-4}, \quad \alpha_{n-i}=-i \lambda_{n-i}+1, \quad \alpha_{n}=-i \lambda_{n}, \\
x_{0 j} & =\frac{U_{j, n}}{U_{1, n}}, \quad x_{i j}=\frac{U_{j, n-4+i}}{U_{1, n-4+i}}-\frac{U_{j, n}}{U_{1, n}}, \\
x_{0 n} & =-\frac{U}{U_{1, n}}=-x_{i n}, \quad x_{01}=1, \\
x_{1 n-3} & =-\frac{U}{U_{1, n-3}}, \quad x_{2 n-2}=-\frac{U}{U_{1, n-2}}, \quad x_{3 n-1}=-\frac{U}{U_{1, n-1}}, \\
& \text { for } i=1,2,3, \quad j=2, \ldots, \quad n-4,
\end{aligned}
$$

with

\footnotetext{
${ }^{4}$ As in [9], we restrict our attention to a subset of the allowed configurations on the celestial sphere, for which the amplitudes can be expressed in terms of generalized hypergeometric functions.
}

$$
\begin{aligned}
U & =\epsilon_{n-3} \epsilon_{n-2} \epsilon_{n-1} \epsilon_{n} \operatorname{det}\left(q_{n-3} q_{n-2} q_{n-1} q_{n}\right), \\
U_{i, j} & =\epsilon_{i} \epsilon_{j} \epsilon_{n-3} \epsilon_{n-2} \epsilon_{n-1} \epsilon_{n} \operatorname{det}\left(q_{n-3} \cdots q_{j \rightarrow i} \cdots\right) .
\end{aligned}
$$

First, notice that the factor $c_{2} / c_{1}$ has poles in $\lambda_{j}$ for $j=3, \ldots, n-4$, so the conformally soft limit of any of these gluons will be given by the residue. Since, as discussed previously, the factor $z_{12}^{3} /\left(z_{23} \cdots z_{n 1}\right)$ gives the appropriate soft factor, these amplitudes obey the conformally soft theorem so long as $\hat{\varphi}_{n}$ becomes independent of $\left(z_{j}, \bar{z}_{j}\right)$ in the limit $\lambda_{j} \rightarrow 0$. The only dependence on $\left(z_{j}, \bar{z}_{j}\right)$ in $\hat{\varphi}_{n}$ appears in $P_{j}^{-i \lambda_{j}}$, which behaves as $P_{j}^{-i \lambda_{j}} \rightarrow 1$ in the conformally soft limit. Hence, $\hat{\varphi}_{n}$ becomes independent of $\left(z_{j}, \bar{z}_{j}\right)$ and the celestial amplitude obeys the conformally soft theorem.

To obtain the conformally soft theorem for gluons $j=n-3, \ldots, n$, we work with an expression for the Mellin-transformed amplitude before the change of variables that gives (A1)

$$
\begin{aligned}
\tilde{\mathcal{A}}_{--+\cdots+}= & \frac{1}{(-2)^{n-4}} \frac{z_{12}^{3}}{z_{23} \cdots z_{n 1}|U|} \prod_{a=1}^{n-4} \int_{0}^{\infty} d \omega_{a} \omega_{a}^{i \lambda_{a}} \frac{\omega_{1} \omega_{2}}{\omega_{3} \cdots \omega_{n-4}} \\
& \times \prod_{b=n-3}^{n}\left(\omega_{b}^{*}\right)^{i \lambda_{b}-1} \Theta\left(\omega_{b}^{*}\right),
\end{aligned}
$$

where

$$
\omega_{j}^{*}=-\frac{1}{U} \sum_{i=1}^{n-4} \omega_{i} U_{i, j}
$$

To take the conformally soft limit on the $n$th leg, we use the identity (2.8) so that the factor $\left(\omega_{n}^{*}\right)^{i \lambda_{n}-1}$ is turned into $\delta\left(\omega_{n}^{*}\right)$. As before, we need to show that the $n$-point amplitude becomes proportional to an $(n-1)$-point amplitude. To this end, we can use $\delta\left(\omega_{n}^{*}\right)$ to perform one of the $\omega_{a}$ integrals. For example, we can solve $\omega_{n}^{*}=0$ for $\omega_{n-4}$,

$$
\omega_{n-4}=-\sum_{i=1}^{n-5} \frac{U_{i, n}}{U_{n-4, n}} \omega_{i},
$$

and perform the integral with respect to $\omega_{n-4}$. We obtain an expression similar to (A5)

$$
\begin{aligned}
\lim _{\lambda_{n} \rightarrow 0} i \lambda_{n} \tilde{\mathcal{A}}_{--+\cdots+}= & \frac{1}{(-2)^{n-4}} \frac{z_{12}^{3}}{z_{23} \cdots z_{n 1}\left|U_{n-4, n}\right|} \\
& \times \prod_{a=1}^{n-5} \int_{0}^{\infty} d \omega_{a} \omega_{a}^{i \lambda_{a}} \frac{\omega_{1} \omega_{2}}{\omega_{3} \cdots \omega_{n-5}} \\
& \times \prod_{b=n-4}^{n-1}\left(\omega_{b}^{*}\right)^{i \lambda_{b}-1} \Theta\left(\omega_{b}^{*}\right),
\end{aligned}
$$

where $\omega_{n-4}^{*}$ is given by the right-hand side of (A7) and 


$$
\begin{aligned}
\omega_{b}^{*} & =-\frac{1}{U} \sum_{i=1}^{n-5}\left(U_{i, b}-\frac{U_{n-4, b} U_{i, n}}{U_{n-4, n}}\right) \omega_{i}, \\
b & =n-3, n-2, n-1 .
\end{aligned}
$$

To recognize that the right-hand side of (A8) is proportional to an $(n-1)$-point amplitude, we put it in the form (A5). Introducing analogues of (A4) where $n-3, \ldots, n$ are replaced with $n-4, \ldots, n-1$,

$$
\begin{aligned}
U^{\prime} & =\epsilon_{n-4} \epsilon_{n-3} \epsilon_{n-2} \epsilon_{n-1} \operatorname{det}\left(q_{n-4} q_{n-3} q_{n-2} q_{n-1}\right), \\
U_{i, j}^{\prime} & =\epsilon_{i} \epsilon_{j} \epsilon_{n-4} \epsilon_{n-3} \epsilon_{n-2} \epsilon_{n-1} \operatorname{det}\left(q_{n-4} \cdots q_{j \rightarrow i} \cdots\right),
\end{aligned}
$$

and using the identity

$$
U_{i, b} U_{n-4, n}-U_{i, n} U_{n-4, b}=-U U_{i, b}^{\prime},
$$

we find (A8) can be written as

$$
\begin{aligned}
\lim _{\lambda_{n} \rightarrow 0} i \lambda_{n} \tilde{\mathcal{A}}_{--+\cdots+}= & -\frac{1}{2} \frac{1}{(-2)^{n-5}} \frac{z_{n-11}}{z_{n-1 n} z_{n 1}} \frac{z_{12}^{3}}{z_{23} \cdots z_{n-11}\left|U^{\prime}\right|} \\
& \times \prod_{a=1}^{n-5} \int_{0}^{\infty} d \omega_{a} \omega_{a}^{i \lambda_{a}} \frac{\omega_{1} \omega_{2}}{\omega_{3} \cdots \omega_{n-5}} \\
& \times \prod_{b=n-4}^{n-1}\left(\omega_{b}^{*}\right)^{i \lambda_{b}-1} \Theta\left(\omega_{b}^{*}\right),
\end{aligned}
$$

where now

$$
\omega_{j}^{*}=-\frac{1}{U^{\prime}} \sum_{i=1}^{n-5} \omega_{i} U_{i, j}^{\prime},
$$

so that the right-hand side of (A12) is readily identified as proportional to an $(n-1)$-point amplitude. The conformally soft theorem of (A5) for the gluons $j=n-3, n-2$, $n-1$ follows from similar arguments.
[1] T. He, P. Mitra, and A. Strominger, 2D Kac-Moody symmetry of 4D Yang-Mills theory, J. High Energy Phys. 10 (2016) 137.

[2] C. Cheung, A. de la Fuente, and R. Sundrum, 4D scattering amplitudes and asymptotic symmetries from 2D CFT, J. High Energy Phys. 01 (2017) 112.

[3] S. Pasterski, S. H. Shao, and A. Strominger, Flat space amplitudes and conformal symmetry of the Celestial sphere, Phys. Rev. D 96, 065026 (2017).

[4] A. Strominger, Lectures on the Infrared Structure of Gravity and Gauge Theory (Princeton University Press, Princeton, 2018).

[5] S. Pasterski and S. H. Shao, Conformal basis for flat space amplitudes, Phys. Rev. D 96, 065022 (2017).

[6] S. Pasterski, S. H. Shao, and A. Strominger, Gluon amplitudes as 2d conformal amplitudes, Phys. Rev. D 96, 085006 (2017).

[7] H. T. Lam and S. H. Shao, Conformal basis, optical theorem, and the bulk point singularity, Phys. Rev. D 98, 025020 (2018).

[8] N. Banerjee, S. Banerjee, S. Atul Bhatkar, and S. Jain, Conformal structure of massless scalar amplitudes beyond tree level, J. High Energy Phys. 04 (2018) 039.

[9] A. Schreiber, A. Volovich, and M. Zlotnikov, Tree-level gluon amplitudes on the celestial sphere, Phys. Lett. B 781, 349 (2018).

[10] S. Banerjee, Null infinity and unitary representation of the Poincare group, J. High Energy Phys. 01 (2019) 205.

[11] S. Stieberger and T. R. Taylor, Strings on Celestial sphere, Nucl. Phys. B935, 388 (2018).

[12] L. Donnay, A. Puhm, and A. Strominger, Conformally soft photons and gravitons, J. High Energy Phys. 01 (2019) 184.
[13] S. Stieberger and T. R. Taylor, Symmetries of Celestial amplitudes, Phys. Lett. B 793, 141 (2019).

[14] S. Banerjee, P. Pandey, and P. Paul, Conformal properties of soft-operators - 1: Use of null-states, arXiv:1902.02309.

[15] W. Fan, A. Fotopoulos, and T. R. Taylor, Soft limits of Yang-Mills amplitudes and conformal correlators, J. High Energy Phys. 05 (2019) 121.

[16] C. Cardona and Y.t. Huang, S-matrix singularities and CFT correlation functions, J. High Energy Phys. 08 (2017) 133.

[17] J. de Boer and S. N. Solodukhin, A holographic reduction of Minkowski space-time, Nucl. Phys. B665, 545 (2003).

[18] G. Barnich and C. Troessaert, Symmetries of Asymptotically Flat 4 Dimensional Spacetimes at Null Infinity Revisited, Phys. Rev. Lett. 105, 111103 (2010).

[19] G. Barnich and C. Troessaert, Aspects of the BMS/CFT correspondence, J. High Energy Phys. 05 (2010) 062.

[20] G. Barnich and C. Troessaert, Supertranslations call for superrotations, Proc. Sci. CNCFG2010 (2010) 010; Ann. Univ. Craiova Phys. 21, S11 (2011).

[21] D. Kapec, V. Lysov, S. Pasterski, and A. Strominger, Semiclassical Virasoro symmetry of the quantum gravity $\mathcal{S}$-matrix, J. High Energy Phys. 08 (2014) 058.

[22] D. Kapec, P. Mitra, A. M. Raclariu, and A. Strominger, 2D Stress Tensor for 4D Gravity, Phys. Rev. Lett. 119, 121601 (2017).

[23] D. Kapec and P. Mitra, A $d$-dimensional stress tensor for Mink $_{d+2}$ gravity, J. High Energy Phys. 05 (2018) 186.

[24] N. Arkani-Hamed, T. C. Huang, and Y.t. Huang, Scattering amplitudes for all masses and spins, arXiv:1709.04891. 
[25] L. Rodina, Scattering Amplitudes from Soft Theorems and Infrared Behavior, Phys. Rev. Lett. 122, 071601 (2019).

[26] D. Nandan, A. Schreiber, A. Volovich, and M. Zlotnikov, Celestial amplitudes: conformal partial waves and soft limits, arXiv:1904.10940.

[27] A. Strominger, On BMS invariance of gravitational scattering, J. High Energy Phys. 07 (2014) 152.

[28] M. Campiglia and A. Laddha, Loop corrected soft photon theorem as a ward identity, arXiv:1903.09133.
[29] G. Veneziano, Construction of a crossing - symmetric, Regge behaved amplitude for linearly rising trajectories, Nuovo Cimento A 57, 190 (1968).

[30] M. B. Green and J.H. Schwarz, Supersymmetrical dual string theory. 2. Vertices and trees, Nucl. Phys. B198, 252 (1982).

[31] D. J. Gross, J. A. Harvey, E. J. Martinec, and R. Rohm, Heterotic string theory. 2. The interacting heterotic string, Nucl. Phys. B267, 75 (1986). 